\title{
The sonographic medical act
}

\author{
Cosima Schiavone ${ }^{1} \cdot$ Roberto Grassi $^{2} \cdot$ Michele Bertolotto $^{3} \cdot$ Carmelo Privitera $^{4}$. Giovanni lannetti ${ }^{5}$. \\ Fabrizio Calliada ${ }^{6} \cdot$ Vito Cantisani $^{7}$
}

Received: 23 July 2020 / Accepted: 24 July 2020 / Published online: 23 September 2020

(c) Società Italiana di Ultrasonologia in Medicina e Biologia (SIUMB) 2020

\section{Introduction}

The Italian Society of Ultrasonography in Medicine and Biology (SIUMB) and the Italian Society of Medical and Interventional Radiology (SIRM) have jointly deemed it appropriate to prepare a document on the sonographic medical act outlining the characteristics of the said act and the physician sonologist's education and competencies. Over the last 10 years, SIUMB and SIRM have organized training activities in the field of diagnostic sonographic imaging.

SIUMB was founded in 1987 and has among its institutional purposes that of stimulating and developing studies of the application of sonography in the field of Medicine and Biology.

Since its foundation in 1913, SIRM has had among its institutional aims the cultural updating of Radiology specialists and training in the study of biomedical imaging, including sonography, in terms of the physical, biological

Cosima Schiavone

cschiavone@unich.it

1 Unit of Internistic Ultrasound, SSD Gastroenterology, Department of Medicine and Science of Aging,

G.D'Annunzio University, Chieti-Pescara, Chieti, Italy

2 Medical-Surgical Department of Clinical and Experimental Internistics "F. Magrassi and A.Lanzara", Faculty Medicineand Surgery, II University of Naples, Naples, Italy

3 U.O.C. Radiology Cattinara Hospital, University of Trieste, Trieste, Italy

4 Complex Operative Unit of Radiology, Vittorio Emanuele Hospital, Catania, Italy

5 UOSD Internistic Ultrasound, Pescara Hospital, Pescara, Italy

6 Department of Clinical Surgical, Diagnostic and Pediatric Sciences, University of Pavia, Pavia, Italy

7 UOS of Diagnostic and Ultrasonographic Innovations, Policlinico Umberto I, University of Rome Sapienza, Rome, Italy and diagnostic features as well as radiation protection and computerization [1].

SIUMB and SIRM have organized-and still organize-numerous joint training activities aimed at the development of sonographic imaging and training of physician sonologists. The two societies represent about 14,000 physicians whose professional activity is clearly defined and documented.

It is, therefore, the responsibility of these two scientific Societies to issue a joint document stating the characteristics of the sonographic medical act and the education of physician sonologists [2].

\section{Rationale}

Preparation of a document dealing with the sonographic medical act was necessary for the following reasons:

- The use of sonographic imaging is not properly regulated in legal terms. This has led to an uncontrolled rise in requests for sonographic imaging services performed by operators whose competence is not clearly defined and documented. The current legislation does not require any specialty training and/or qualification to carry out a sonographic examination.

- There is some confusion about the very concept of the sonographic medical act related both to the operator's competence and education and to the basic characteristics of the sonographic examination in whose absence the examination is to be considered incomplete or incorrectly performed.

\section{Definition of the sonographic medical act}

A sonographic medical act is defined as an independent procedure performed by a physician sonologist holding a license to practise the medical profession and who uses sonography 
as a tool to make a diagnosis or to carry out interventional or therapeutic procedures on the basis of a clinical question formulated by him/herself or by another physician [3].

Despite the terminological confusion, the sonographic medical act is different from other forms of use of sonography, such as a contribution to another medical act or paramedic healthcare service. The sonographic medical act is therefore different from sonographic support (office sonography, echoscopy, etc.) which is an integration of sonography into another examination.

In sonographic support, the medical act (if the examination is carried out by a qualified physician) or the paramedical performance (e.g. an obstetric examination), the essence is not the use of sonography, but the examination itself in which the use of sonography is only a part. It follows that the way the ultrasound scanner is used must necessarily be different, like the obligation to report: if an examination involves sonographic support, the report will document the result of the examination as a whole including the data obtained using sonographic evaluation. In this report, the terms "sonography" and "sonographic medical act" will be used interchangeably. The "sonographic support" will not be mentioned.

\section{The physician sonologist's education}

Sonography is part of the university training path of numerous specialty schools. The sonographic medical act is thus not exclusively carried out by radiology specialists, as it is within the competence of all specialists who have attended an appropriate theoretical-practical training programme. Sonography falls within the competence of any qualified physician, once he/she has completed his/her education and acquired the necessary skills and has correctly performed a suitable number of sonographic examinations of different types under the supervision of accredited tutors. However, sonography is exclusively a medical act as the images are interpreted for diagnostic and therapeutic purposes, which is a specific activity carried out by qualified physicians. It cannot be delegated to non-physician health professionals nor to physicians who have not personally carried out the sonographic evaluation.

\section{Characteristics of the sonographic medical act}

The sonographic medical act is divided into a series of interdependent operational and/or decision-making phases:

- Analysis of the request for an examination;

- The patient's clinical and medical history;
- Justification for the proposed examination;

- Informed consent acquisition;

- Facultative physical examination

- Sonographic examination;

- Interpretation and reporting;

- Communication of the diagnosis.

In this form, the sonographic medical act benefits in all phases of the examination from the physician sonologist's professional contribution, i.e. before the examination, the manoeuvres required to obtain the images, the iconographic documentation, evaluation of the exhaustiveness of the investigation and/or the necessity to propose further diagnostic imaging or further diagnostic-instrumental investigation.

\section{Analysis of the request for an examination}

The physician sonologist must examine the request for an evaluation by taking note of the clinical question, the type of sonographic investigation deemed appropriate and the degree of urgency. The request for investigation forwarded by the prescribing physician is a "proposal", not a "binding prescription". It is up to the physician sonologist to confirm the indication for the examination.

\section{The patient's clinical and medical history}

The physician sonologist must receive the details regarding the patient's condition that may allow him/her to correctly classify the clinical case. If the prescribing physician has provided incomplete information, at least the essential information must be collected.

\section{Justification for the proposed examination}

The sonographic medical act is justified if it presents the possibility to acquire useful information able to influence the subsequent diagnostic/therapeutic process. The justification is therefore closely linked to the knowledge of the patient's clinical situation. It is also necessary to consider the technology availability, the experience and competence of the operator and the organizational context.

\section{Informed consent acquisition}

In common medical practice, consent does not necessarily have to be expressed in writing. Whatever the form, it is essential that it is an adequately informed consent based on information provided by the physician. In the field of sonography, consent can be implicit or tacit in case of common, non-invasive examinations, but explicit informed written consent is required, if the diagnostic-therapeutic procedure involves a risk (e.g. interventional or contrast-enhanced 
procedures). It is the responsibility of the physician sonologist to obtain informed consent.

\section{Physical examination}

Before ultrasound examination is performed, the medical doctor may evaluate the patients with a physical examination in case of specific conditions in order to have better clinical clues and properly address the examination.

\section{Sonographic examination}

The sonographic examination is a complex act which makes it necessary for the physician sonologist to use the sonographic tool for the time required to collect the necessary diagnostic information or perform a therapeutic procedure. The physician sonologist must first of all evaluate the adequacy of the equipment, choose the probe and optimize the scan parameters. He/she must subsequently scan the organs using an appropriate technique and evaluate the diagnostic quality of the images.

The iconographic documentation and accompanying report are essential to a correct sonographic medical act. The physician sonologist must adhere to the minimum criteria of iconographic documentation required to provide a complete documentation also with a view to future reference or possible re-evaluation. All conditions limiting the diagnostic quality of the examination, such as anatomical, physiological or pathological causes must be reported. The iconographic documentation, in printed or digital form, must be kept in accordance with legal requirements.

\section{Interpretation and reporting}

The report is the most significant and relevant element of the sonographic medical act as it provides the diagnosis made on the basis of the interpretation of the images. The report is mandatory as it describes the professional performance carried out by the physician sonologist. Interpretation of the images occurs mainly during the examination while searching for information on clinical/diagnostic interest, subsequently summarized in the report. The report is a public deed as well as a document of medico-legal responsibility which must be filed in accordance with legal requirements.

\section{Communication of the diagnosis}

Communication of the outcome concludes the sonographic examination and concerns first of all the patient, whose health is under examination, and the attending physician, if he/she is not the physician sonologist.

In outpatient services, the first person to receive the report is the patient; if the patient is hospitalized, the patient him/herself and the physician in charge of the hospital ward care will be the first to receive the report. Particular attention should be paid to those patients who present complex problems as the outcome must be communicated effectively and truthfully but with due respect for the patient's feelings. Timeliness of communication constitutes an element of possible medico-legal responsibility. If damage occurs because the outcome is not promptly communicated, the physician sonologist may be held responsible for the omission.

\section{Conclusions}

Sonography is an important medical act carried out within the framework of the ethical and deontological standards of the medical profession. It is a professional act aimed at promoting health, preventing diseases, diagnosing and prescribing therapies or rehabilitative treatments to patients, individuals, groups or communities. Sonographic examination performed as a medical act is the responsibility of a qualified physician. It is a complex medical procedure requiring clinical and technical skills obtainable only through an educational process which includes certification of acquired skills.

Like all other diagnostic imaging examinations, sonography can be subject to the risk of errors which may occur during the examination and/or interpretation of the images, and it may, therefore, involve medico-legal responsibilities. A report which is perfect from a formal point of view can be the best medico-legal protection. In addition to the quality of the examination, the physician sonologist must also be able to demonstrate his/her professional competence and expertise. Reporting and performance of the examination cannot be separated from each other in the sonographic medical act as sonography is a dynamic examination summarized in the report, based on a set of considerations not limited to the evaluation of the images.

\section{References}

1. Brunese L, Fileni A, Tamburrini O (2012) L'atto medico ecografico dello specialista in Radiodiagnostica. https://www.sirm. org/wp-content/uploads/2020/05/AttoMedico_Ecografico.pdf. Accessed 20 July 2020

2. SIRM-SIUMB (2018) L'atto medico ecografico. https://www. siumb.it/files/doc/ATTO\%20MEDICO\%20ECOGRAFICO_ SIRM_SIUMB_23.04.2018.pdf. Accessed 20 July 2020

3. European Union of Medical Specialists (2008) UEMS 2008/03 FINAL. https://www.uems.eu/_data/assets/pdf_ file/0008/1430/960.pdf. Accessed 20 July 2020

Publisher's Note Springer Nature remains neutral with regard to jurisdictional claims in published maps and institutional affiliations. 